\title{
The Extension of the Van Der Pauw Method to Anisotropic Media
}

\author{
Jonas KLEIZA \\ Vilnius Technical University \\ Sauletekio al. 11, 10223 Vilnius, Lithuania \\ e-mail:kleiza@mail.tele2.lt \\ Mifodijus SAPAGOVAS, Vytautas KLEIZA \\ Institute of Mathematics and Informatics \\ Akademijos 4, 08663 Vilnius, Lithuania \\ e-mail:mifosap@ktl.mii.lt,vytautas.kleiza@ktl.mii.lt
}

Received: November 2006

\begin{abstract}
The method for calculating the specific conductivity tensor of an anisotropically conductive medium, proposed in this paper, distinguishes itself by the simplicity of physical measurements: it suffices to make an equally thick rectangle-shaped sample with four electrodes fixed on its sides and to take various measurements of current intensity and differences of potentials. The necessary mathematical calculations can be promptly performed, even without using a complex computing technique. The accuracy of the results obtained depends on the dimensions of the sample and on the ratios of the conductivity tensor components.
\end{abstract}

Key words: modelling, anisotropic media, electrical conductivity, current flow, numerical methods, measurements.

\section{Introduction}

Thin layer structures are wide used in the contemporary microelectronic technique devices, therefore investigations of the later by solid body physical methods acquire ever more important significance (Qiu, 2002). Thin layers of ordered structure (first of all monocrystalline) are of particular importance, since many physical effects in such layers distinguish themselves by a good repeatability. Thin films of ordered structure, obtained by epitaxy methods (by growing a layer on a monocrystalline substrate or inducing crystallite orientation by an electric field acting in the plane of the substrate), most frequently are anisotropic relative to electric conductivity, therefore their specific electric conductivity is completely defined by a tensor.

The van der Pauw (VdP) structure, based upon the theoretical work of van der Pauw (Pauw, 1958), is a test structure widely used for measuring resistivity of arbitrary shaped samples of constant thickness. Over the years, many researchers have extended the original ideas to develop a variety of approaches for evaluating the resistivities of both 
isotropic an anisoptropic materials using the VdP type structures (Futamata, 1992; Ramadan, 1994; Gelder, 1995; Rietveld, 2003).

This paper deals with various methods for defining an electrical conductivity tensor of an anisotropic medium. Application of the van der Pauw theorem and equation makes the basis of all of them. This equation is well known in the Hall effect theory and is meant for establishing the conductivity of an isotropic medium. Its coefficients are found by measuring differences of potentials and intensity of current.

\section{The General Electrical Conductivity of the Anisotropic Medium and its Calculation by the van der Pauw Method}

\subsection{Boundary Problem of Potential Distribution in the Anisotropic Medium and its Transformation}

Distribution of the electric field potential $\phi(x, y)$ in a plain domain $G$, electrical conductivity of which is defined by the tensor

$$
\sigma=\left(\begin{array}{ll}
\sigma_{11} & \sigma_{12} \\
\sigma_{12} & \sigma_{22}
\end{array}\right), \quad d=\operatorname{det} \sigma=\sigma_{11} \sigma_{22}-\left(\sigma_{12}\right)^{2}>0
$$

is the solution to the boundary value problem

$$
\left\{\begin{array}{l}
\operatorname{divj}=0, \quad(x, y) \in G, \\
\mathbf{j}=\sigma \operatorname{grad} \varphi \\
\left.\varphi\right|_{\kappa}=\text { const } \\
\left.(\sigma \operatorname{grad} \varphi)_{n}\right|_{\Gamma \backslash \kappa}=0 .
\end{array}\right.
$$

Here $\Gamma$ is the contour of domain $G, \kappa$ is the part of the contour that is coincident with the contacts $\kappa_{1}, \kappa_{2}, \ldots, \kappa_{i}$, and $n$ is the contour normal (see Fig. 1a).

After replacing variables in problem (2) by

$$
\xi=x \sigma_{22}-y \sigma_{12}, \quad \eta=y \sqrt{d},
$$

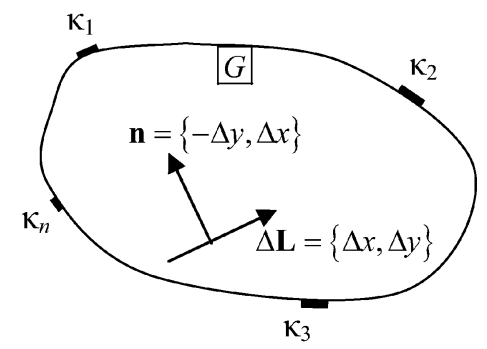

a)

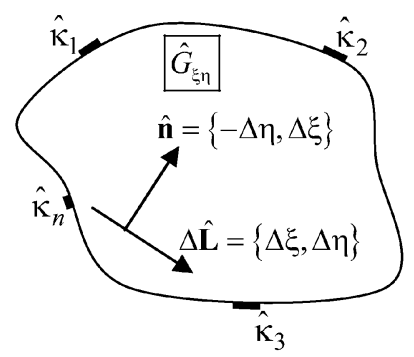

b)

Fig. 1. Calculation of the amount of current flowing through the elementary segment. 
we have a boundary problem (Zilinskas, 1982) for the function $\hat{\varphi}(\xi, \eta)=\varphi(x, y)$ in the respective domain $\widehat{G}$ of the Laplace equation:

$$
\left\{\begin{array}{l}
\operatorname{div} \hat{\mathbf{j}} \equiv \Delta \hat{\varphi}(\xi, \eta)=0, \quad(\xi, \eta) \in \hat{G} \\
\hat{\mathbf{j}}=\operatorname{sgrad} \hat{\varphi} \\
\left.\hat{\varphi}\right|_{\hat{\kappa}}=\text { const } \\
\left.\frac{\partial \hat{\varphi}}{\partial \hat{n}}\right|_{\hat{\Gamma} \backslash \hat{\kappa}}=0
\end{array}\right.
$$

Here $\hat{n}_{\xi \eta}$ is the contour normal of domain $\widehat{G}$.

In the physical sense, (4) defines the potential distribution in an isotropic plate $\widehat{G}$. We prove the statement that allows us to establish its specific conductivity $s$.

Theorem. If $s=\sqrt{\operatorname{det} \sigma}$ in problem (4), then problems (3) and (4) are equivalent.

Proof. We investigate the amount of current $\Delta I_{x y}$ flawing through the elementary segment $\Delta L$ of the domain $G$, that is defined by the vector $\Delta \mathbf{L}=\{\Delta x, \Delta y\}$ :

$$
\begin{aligned}
\Delta I_{x y} & =\left.(\sigma \operatorname{grad} \varphi)\right|_{n}|\Delta \mathbf{L}|=\left\{\sigma_{11} \varphi_{x}+\sigma_{12} \varphi_{y}, \sigma_{12} \varphi_{x}+\sigma_{22} \varphi_{y}\right\} \cdot\{-\Delta y, \Delta x\} \\
& =\left(\sigma_{12} \varphi_{x}+\sigma_{22} \varphi_{y}\right) \Delta x+\left(-\sigma_{11} \varphi_{x}-\sigma_{12} \varphi_{y}\right) \Delta y
\end{aligned}
$$

The image of the vector $\Delta \mathbf{L}$ in the system of coordinates $(\xi, \eta)$ is

$$
\Delta \hat{\mathbf{L}}=\{\Delta \xi, \Delta \eta\}=\left\{\sigma_{22} \Delta x-\sigma_{12} \Delta y, \sqrt{d} \Delta y\right\},
$$

and the amount of current $\Delta I_{\xi \eta}$ flowing through the respective segment $\Delta \hat{L}$ is equal to

$$
\begin{aligned}
\Delta I_{\xi \eta} & =s \frac{\partial \hat{\varphi}}{\partial \hat{n}}|\Delta \hat{\mathbf{L}}|=s\left(-\hat{\varphi}_{\xi} \Delta \eta+\hat{\varphi}_{\eta} \Delta \xi\right) \\
& =s\left(-\hat{\varphi}_{\xi} \sqrt{d} \Delta y+\hat{\varphi}_{\eta}\left(\sigma_{22} \Delta x-\sigma_{12} \Delta y\right)\right) .
\end{aligned}
$$

Since $\hat{\varphi}_{\xi}=\varphi_{x} / \sigma_{22}, \hat{\varphi}_{\eta}=\varphi_{x} \sigma_{12} /\left(\sigma_{22} \sqrt{d}\right)$, we have

$$
I_{\xi \eta}=s\left(-\varphi_{x} \frac{\sqrt{d}}{\sigma_{22}} \Delta y+\left(\varphi_{x} \frac{\sigma_{12}}{\sigma_{22}} \sqrt{d}+\frac{\varphi_{y}}{\sqrt{d}}\right)\left(\sigma_{22} \Delta x-\sigma_{12} \Delta y\right)\right) .
$$

By comparing the coefficients at $\varphi_{x} \Delta x, \varphi_{x} \Delta y, \varphi_{y} \Delta x, \varphi_{y} \Delta y$ in expressions (5) and (6), we get that $s=\sqrt{d}=\sqrt{\operatorname{det} \sigma}$.

1.2. Application of the van der Pauw Method in Finding the General Conductivity of an Anisotropic Medium

In 1958, van der Pauw (Pauw, 1958) proposed a method for measuring the specific electrical conductivity of a sample of any geometrical form. To realize this method, a constant 


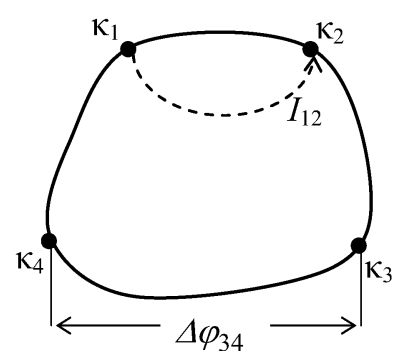

a)

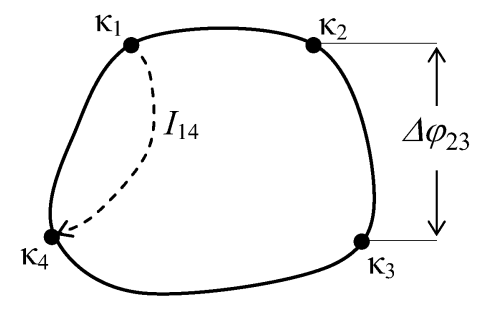

b)

Fig. 2. Measurement of current and potential differences in van der Pauw experiments.

thickness sample $h$ was used with 4 point contacts that are arranged in the sample perimeter. In order to calculate specific conductivity, first we induce voltage between the contacts $\kappa_{1}, \kappa_{2}$, and measure the intensity $I_{12}$ of the flowing current as well as the difference $\Delta \varphi_{34}$ of potentials appearing between the remaining contacts (Fig. 2a). Afterwards, the experiment is repeated, by inducing the equivalent voltage between the contacts $\kappa_{1}, \kappa_{4}$ and measuring the current $I_{14}$ as well as the difference $\Delta \varphi_{23}$ of potentials (Fig. 2b).

Van der Pauw has proved that the specific conductivity $s$ of the sample is the solution to the equation

$$
\exp \left(-\pi h s\left|\Delta \varphi_{34}\right| / I_{12}\right)+\exp \left(-\pi h s\left|\Delta \varphi_{23}\right| / I_{14}\right)=1
$$

This equation can be solved uniquely and its solution $s$ can be found by the Newton method, selecting any initial approximation $s^{(0)}$, e.g., $s^{(0)}=0$.

Thus, if the sample conductivity is anisotropic, defined by the tensor $\sigma(1)$, then, on the basis of equivalence of problems (3) and (4), we obtain that the solution to equation (7) $s=\sqrt{\operatorname{det} \sigma}$.

\section{Formulation and Solution of the Main Problems}

Let the form of an anisotropically conductive sample be a rectangle $G$ whose sides are arbitrarily oriented with respect to the main directions of a tensor. On the two opposite sides of the rectangle the current contacts $\kappa_{1}$ and $\kappa_{3}$ located, while the point contacts $\kappa_{2}$ and $\kappa_{4}$ are on the other sides (Fig. 3). We prove the statements of the following two problems:

Q - to find the potential distribution in the contour of domain $G$ and the intensity $I_{13}$ of current flowing via the contacts $\kappa_{1}$ and $\kappa_{3}$, the conductivity tensor $\sigma$ being known;

$Q^{-1}$ - to establish the conductivity tensor $\sigma$ of a sample, as the current intensity $I_{13}$, potential of the contact $\kappa_{2}$ or $\kappa_{4}$, and the general conductivity $s=\sqrt{\operatorname{det} \sigma}$ are known. 


\subsection{Problem Q: Calculation of the Potential at the Point of Contour and of the Current}

Suppose that the conductivity tensor $\sigma$ is known. Let us rearrange boundary problem (2.) into (3), and making use of the fact that the domain $G$ is a parallelogram (Fig. 3) and Christoffel-Schwarz integral

$$
\xi+i \eta=\mu \int_{0}^{z} f_{\alpha, k}(\tau) \mathrm{d} \tau
$$

conformally maps the half-plane $z=t+q i,(q \geqslant 0)$ into it. Here

$$
f_{\alpha, k}(\tau)=\tau^{\alpha-1}(1-\tau)^{-\alpha}(1-k \tau)^{\alpha-1}, \quad \mu=a \sigma_{22}\left(\int_{0}^{1} f_{\alpha, k}(\tau) \mathrm{d} \tau\right)^{-1}
$$

Based on the conformability of mapping (8.) (Kleiza, 1992), we get a parametric expression of the potential in the side $y=0(0 \leqslant x \leqslant a)$ of the rectangle $G$

$$
\left\{\begin{array}{l}
\varphi=V \int_{0}^{t} f_{0.5, k}(\tau) \mathrm{d} \tau / A_{0.5, k} \\
x=a \int_{0}^{t} f_{\alpha, k}(\tau) \mathrm{d} \tau / A_{\alpha, k}
\end{array}\right.
$$

where

$$
t \in[0,1], \quad A_{\alpha, k}=\int_{0}^{1} f_{\alpha, k}(\tau) \mathrm{d} \tau
$$
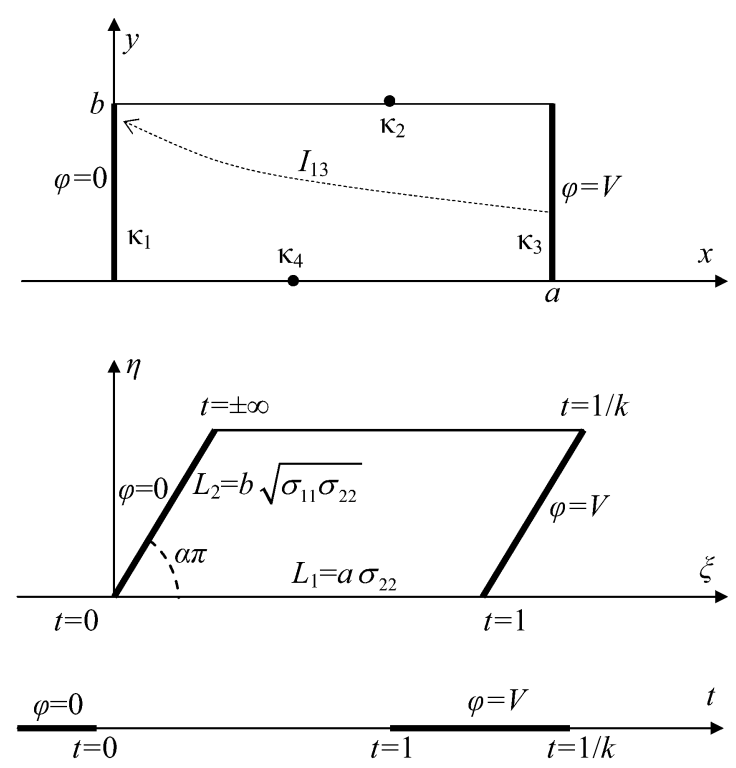

Fig. 3. Mapping of a rectangular form sampe into parallelogram and a half-plane. 
and the parameter $k$ is the solution of the uniquely solvable equation

$$
\alpha=\frac{1}{\pi} \arccos \frac{-\sigma_{12}}{\sqrt{\sigma_{11} \sigma_{22}}}
$$

(because $A_{\alpha, k}$ is an increasing function of the argument $k$ ). This equation was solved after calculating the ratio $L_{2} / L_{1}$ of adjacent side lengths of the parallelogram $\widehat{G}_{\xi \eta}$ and using the equality

$$
\int_{1}^{1 / k}\left|f_{\alpha, k}(\tau)\right| \mathrm{d} \tau=\int_{0}^{1}\left|f_{\alpha, 1-k}(\tau)\right| \mathrm{d} \tau
$$

Having calculated the value of $k$, one can also gets the value of current $I_{13}$ flowing via the sample:

$$
I_{13}=V h \sqrt{\operatorname{det} \sigma} \frac{A_{0.5,1-k}}{A_{0.5, k}}
$$

\subsection{Problem $Q^{-1}$ : Calculation of the Conductivity Tensor $\sigma$}

On the basis of relations (10)-(12), we can obtain the following expressions of tensor components:

$$
\sigma_{11}=\lambda \frac{a}{b} \frac{A_{\alpha, 1-k}}{A_{\alpha, k}}, \quad \sigma_{12}=-\lambda \cos (\alpha \pi), \quad \sigma_{22}=\lambda \frac{b}{a} \frac{A_{\alpha, k}}{A_{\alpha, 1-k}},
$$

where $\lambda=\sqrt{\operatorname{det} \sigma} / \sin (\alpha \pi)$.

Thus, the problem of calculating the tensor $\sigma$ is reduced to determining the numbers $\alpha, k$, and $\sqrt{\operatorname{det} \sigma}$. Assuming that $\sqrt{\operatorname{det} \sigma}$ is known, we will prove that, after physical measurements of the current intensity $I_{13}$ as well as of the potential of contact $\kappa_{2}$ or $\kappa_{4}$, we shall also be able to calculate the parameters $\alpha$ and $k$.

1. The value of the current $I_{13}$ flowing via the plate and that of $\sqrt{\operatorname{det} \sigma}$ being known, the number $k$ can be found by equation (12). This equation can be solved uniquely in respect of the single unknown $k \in(0,1)$, because the integral $A_{0.5, k}$ is function of the argument $k$ increasing from 0 to $\infty$.

2. Let the value of $k$ be known. We shall prove that after measuring the potential $\bar{\varphi}=\varphi(\bar{x}, 0)$, where $\bar{x}$ is the abscissa of the contact $\kappa_{2}$, one can calculate the value of the remaining unknown $\alpha$.

To this end, we consider system (9). Its first equation, as $\varphi=\bar{\varphi}$, is solved uniquely with respect to $t$, because the right-hand side of the equation is a monotone function of the variable $t$, while the integrand function $f_{0.5, k}(\tau)>0$. We shall prove that the second equation of system (9) also has a unique solution $\alpha$. It suffices to prove the monotonicity of the ratio

$$
\bar{x}=\int_{0}^{t} f_{\alpha, k}(\tau) \mathrm{d} \tau / A_{\alpha, k}
$$


of integrals with respect to the variable $\alpha$. After calculating

$$
\frac{\partial^{2} \bar{x}}{\partial t \partial \alpha}=\frac{f_{\alpha, k}(\tau)}{\left(A_{\alpha, k}\right)^{2}}\left(A_{\alpha, k}\left(\ln \left(t-k t^{2}\right)-\ln (t-1)\right)-\frac{\partial A_{\alpha, k}}{\partial \alpha}\right),
$$

we see that the derivative has only one zero in the interval $t \in(0,1)$, because the difference $\ln \left(t-k t^{2}\right)-\ln (1-t)$ varies increasing from $-\infty(t=0)$ up to $+\infty(t=1)$, and the function $f_{\alpha, k}(t)=\tau^{\alpha-1}(1-t)^{-\alpha}(1-k t)^{\alpha-1}>0, t \in(0,1)$. In addition,

$$
\left.\frac{\partial \bar{x}}{\partial \alpha}\right|_{t=0}=\left.\frac{\partial \bar{x}}{\partial \alpha}\right|_{t=1}=0
$$

because

$$
\left.\bar{x}\right|_{t=0} \equiv 0,\left.\quad \bar{x}\right|_{t=1} \equiv 1
$$

Therefore $\frac{\partial \bar{x}}{\partial \alpha}<0, t \in(0,1), \alpha \in(0,1)$ and system (9) is solved uniquely.

Note that $\varphi(x, 0)+\varphi(a-x, b) \equiv V, \forall x \in[a, b]$ therefore the case, where the potential is measured in the side $y=b$, is reduced to that considered above.

The empyrical method for the calculation of unknown parameters of differential equation has been developed in the paper Baronas et al. (2002).

\section{Solution of the Problem Using a Single Sample}

Basing on the statements proved, we are able to calculate the components of a conductivity tensor, using two samples: the firs one - if any shape with 4 point contacts (the general conductivity $\sqrt{\operatorname{det} \sigma}$ is calculated by the van der Pauw method (see Section 1)), and the second one is shaped (determination of a tensor (see Section 2)). Note that when doing physical experiments, it is more convenient to use one sample, and, by respectively changing the contacts the electric current is flowing through to make the necessary measurements of the flowing currents and potentials.

Fig. 4 illustrates succession of the measurements taken and mathematical calculations using a single sample: $\boldsymbol{V} \boldsymbol{d P}$ is the van der Pauw method (see Section 1) to establish the general conductivity, and $Q^{-1}-$ to find the tensor $\sigma^{(0)}$ (see Section 2). It is clear that, due to the finite (non zero) lengths of contacts, the tensor $\sigma^{(0)}$ calculated in this way will differ from the specific sample substance conductivity tensor $\sigma$. We evaluate the value of an error after defining a relative error:

$$
\delta=\frac{\left\|\sigma-\sigma^{(0)}\right\|}{\left\|\sigma^{(0)}\right\|} \cdot 100 \%=\frac{\sqrt{\left(\sigma_{11}-\sigma_{11}^{(0)}\right)^{2}+\left(\sigma_{12}-\sigma_{12}^{(0)}\right)^{2}+\left(\sigma_{22}-\sigma_{22}^{(0)}\right)^{2}}}{\sqrt{\left(\sigma_{11}^{(0)}\right)^{2}+\left(\sigma_{12}^{(0)}\right)^{2}+\left(\sigma_{22}^{(0)}\right)^{2}}} \cdot 100 \% .
$$

Since $\operatorname{det} \sigma>0$, the relation

$$
\sigma=\left(\begin{array}{cc}
\cos \theta & -\sin \theta \\
\sin \theta & \cos \theta
\end{array}\right)\left(\begin{array}{cc}
\sigma_{1} & 0 \\
0 & \sigma_{2}
\end{array}\right)\left(\begin{array}{cc}
\cos \theta & \sin \theta \\
-\sin \theta & \cos \theta
\end{array}\right)
$$


Experiments 1, 2

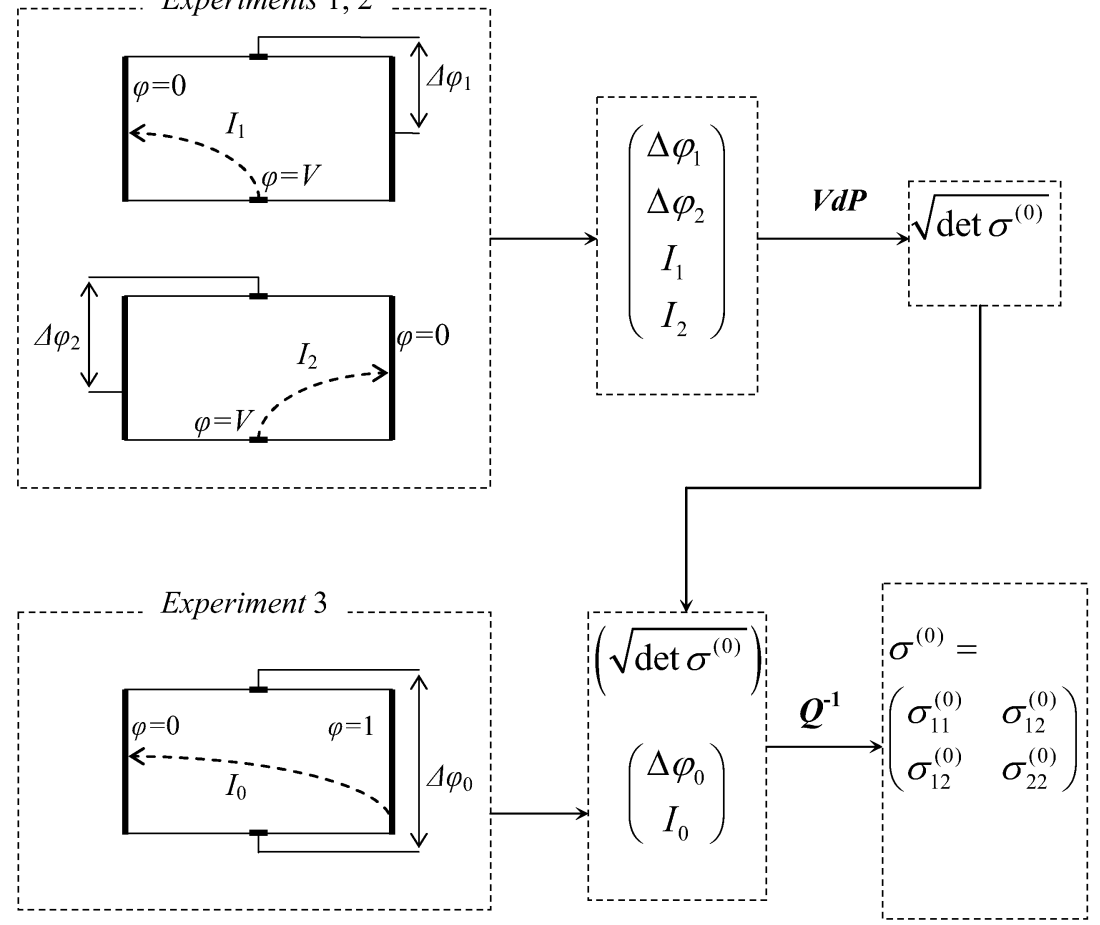

Fig. 4. A diagram for calculating the tensor $\sigma$, using a single rectangular form sample.

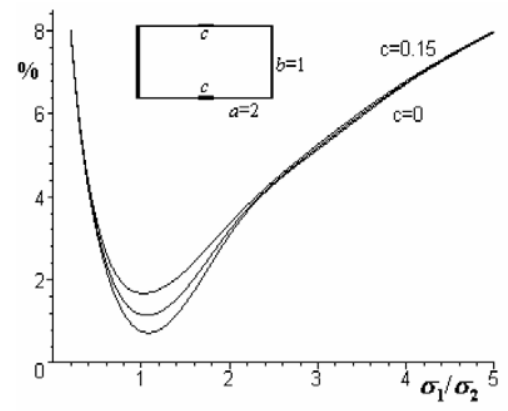

a)

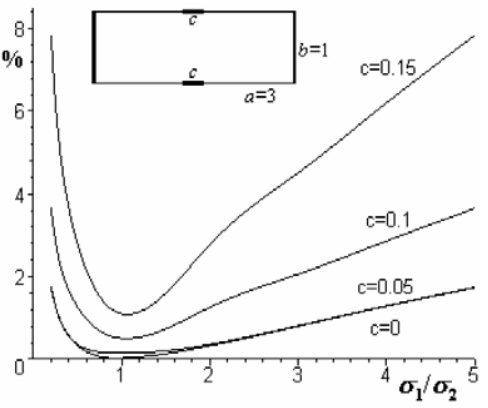

b)

Fig. 5. Distribution of relative errors dependent on the ratio of the main tensor components $\sigma_{1} / \sigma_{2}$, the sample dimensions being $2 \times 1(a)$ and $3 \times 1(b)$.

is always valid. Therefore, with a view to encompass a greater amount of various tensor $\sigma$, Fig. 5 represents the maximal values $\max _{0 \leqslant \theta \leqslant \pi} \delta$, with respect to $\theta$, of the relative errors $\delta$, dependent on the ratio $\sigma_{1} / \sigma_{2}$ of the basic summands, the ratio $a / b$ between length and height of the sample, and on the length of the central contact $c$.

The analysis of these errors shows that or $a / b=2$, the error $\delta$ almost does not depend 
on the $c=0,0.05,0.1,0.15,0.05,0.1,0.15$ (Fig. 5a), and it is minimal as $\sigma_{1}=\sigma_{2}$. If we extend the form of the sample up to 3 (Fig. $5 \mathrm{~b}$ ), we observe a general decrease of error that is particularly vivid for short lengths of the central contact $c=0,0.005,0.1$. As $c=0.15$, the values of errors remain the same in fact in both cases. Thus, applying the method described here in practice, in most frequent cases $0.2 \leqslant \sigma_{1} / \sigma_{2} \leqslant 5, a / b \leqslant$ $3, c \leqslant 0$, the relative error $\delta$ does not exceed $8 \%$.

In the next section, we shall show the procedure after which the errors can be considerably diminished.

\section{The Way of Improving the Solution $\sigma_{11}, \sigma_{12}, \sigma_{22}$}

Let the approximation $\sigma^{(0)}$ of a conductivity tensor be calculated by the method indicated in Section 3. We shall show that, after some mathematical operations, it can be specified.

Let us map (as previously (3.), a rectangle into a parallelogram

$$
\left\{\begin{array}{l}
\xi=x \sigma_{22}^{(0)}-y \sigma_{12}^{(0)} \\
\eta=y \sqrt{\operatorname{det} \sigma^{(0)}}
\end{array}\right.
$$

and its contour into the real axis $t$ of a complex half-plane

$$
t \rightarrow \xi+i \eta=\mu \int_{0}^{t} f_{\alpha, k} \mathrm{~d} \tau
$$

here

$$
f_{\alpha, k}(\tau)=\tau^{\alpha-1}(1-\tau)^{-\alpha}(1-k \tau)^{\alpha-1}, \quad \mu=a \sigma_{22}^{(0)}\left(\int_{0}^{1} f_{\alpha, k} \mathrm{~d} \tau\right)^{-1} .
$$

Since, under the assumption, $\sigma^{(0)}$ in known, one can find the numbers $\alpha$ and $k$ from relations (9), and mapping of the boundary points $t_{i j}$ (i.e., $t_{11}, t_{12}, t_{21}, t_{22}$, see Fig. 4.1) of contacts-according to (14), based on their known coordinates $\xi_{i j}+i \eta_{i j}$ on the sides of the parallelogram. Let us form a function

$$
F_{\alpha}=\frac{\left(T_{1}-\tau\right)\left(T_{2}-\tau\right)}{\sqrt{\tau\left(t_{11}-\tau\right)\left(t_{12}-\tau\right)(1-\tau)(1-k \tau)\left(t_{21}-\tau\right)\left(t_{22}-\tau\right)}} .
$$

Then the integral

$$
t \rightarrow u+i v=\int_{0}^{t} F_{\alpha} \mathrm{d} \tau
$$

will map the real axis $t$ into a decagon $W$ (a rectangle with two cuts, see Fig. 6), in case the numbers $T_{1}$ and $T_{2}$ are chosen so that 

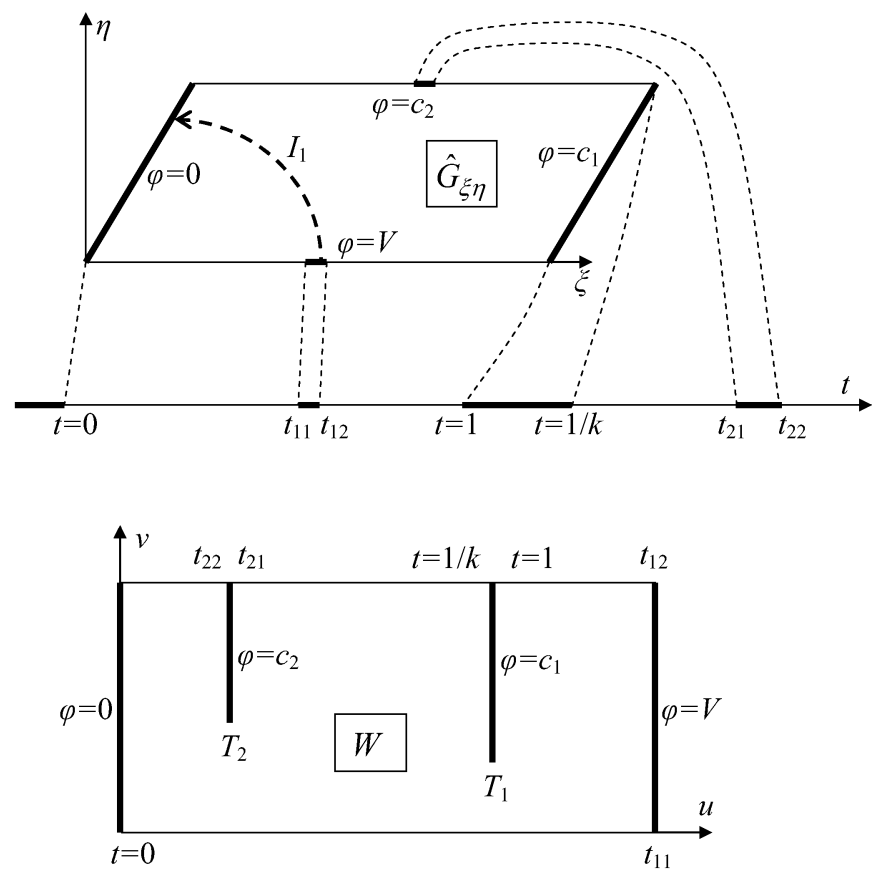

Fig. 6. A scheme of mapping the parallelogram into the decagon.

$$
\int_{1}^{1 / k} F_{\alpha} \mathrm{d} \tau=0, \quad \int_{t_{21}}^{t_{22}} F_{\alpha} \mathrm{d} \tau=0
$$

i.e., on condition that amounts of current flowing through the contacts $\kappa_{2}, \kappa_{3}$ were equal to zero. Since the mapping of (15) is conformal, the conductivity of domain $W$ remains the same $(\sqrt{\operatorname{det} \sigma})$. More over, the potential in it is linearly distributed, so we can calculate the difference of potentials $\Delta \varphi_{1}=c_{1}-c_{2}$ and intensity of current $I_{1}$ :

$$
\Delta \varphi_{1}=V \frac{\int_{1 / k}^{t_{21}} F_{\alpha} \mathrm{d} \tau}{\int_{0}^{t_{11}} F_{\alpha} \mathrm{d} \tau}, \quad I_{1}=V h \sqrt{\operatorname{det} \sigma} \frac{\int_{t_{11}}^{t_{12}}\left|F_{\alpha}\right| \mathrm{d} \tau}{\int_{0}^{t_{11}} F_{\alpha} \mathrm{d} \tau} .
$$

If the current is flowing between other contacts (Fig. 4, the case of current $I_{2}$, we obtain by replacing in mapping (15) the parameter $\alpha$ by $1-\alpha$ ) formulas analogous to (16):

$$
\Delta \varphi_{2}=V \frac{\int_{1 / k}^{t_{21}} F_{1-\alpha} \mathrm{d} \tau}{\int_{0}^{t_{11}} F_{1-\alpha} \mathrm{d} \tau}, \quad I_{2}=V h \sqrt{\operatorname{det} \sigma} \frac{\int_{t_{11}}^{t_{12}}\left|F_{1-\alpha}\right| \mathrm{d} \tau}{\int_{0}^{t_{11}} F_{1-\alpha} \mathrm{d} \tau} .
$$

Consequently, if we know an approximate conductivity tensor $\sigma^{(0)}$, we can calculate the intensity of currents $I_{1}^{(0)}, I_{2}^{(0)}$ (problem $\mathbf{Q}$ ) as well as the corresponding differences of potentials $\Delta \varphi_{1}^{(0)}, \Delta \varphi_{2}^{(0)}$. Then, using them as experimental data and solving the van 


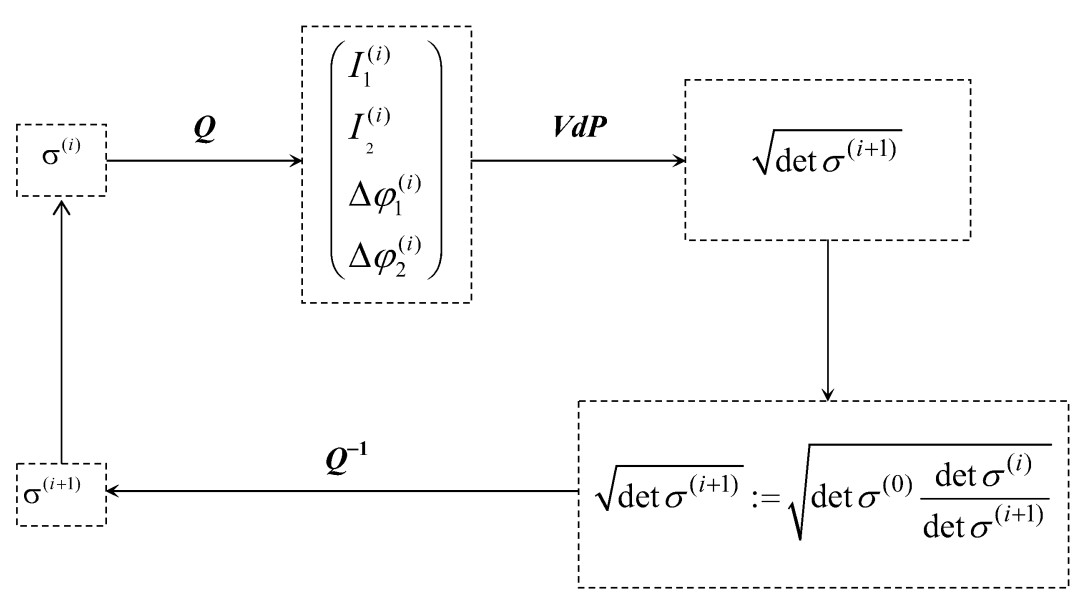

Fig. 7. A diagram of specifying the tensor components.

der Pauw equation, we establish a new conductivity value $\sqrt{\operatorname{det} \sigma^{(1)}}$. The ratio of conductivities

$$
f^{(0)}=\frac{\sqrt{\operatorname{det} \sigma^{(0)}}}{\sqrt{\operatorname{det} \sigma^{(1)}}}
$$

defines the error of the van der Pauw method. Therefore, by assigning the new value $\sqrt{\operatorname{det} \sigma^{(1)}}=\sqrt{\operatorname{det} \sigma^{(0)}} f^{(0)}$ to the tensor determinant, we also determine a specified conductivity tensor $\sigma^{(1)}$ (just like on Section 2). Replacing now the tensor $\sigma^{(0)}$ by $\sigma^{(1)}$, we repeat the calculations described above. We continue this process as long as $f^{(i)}=$ $\frac{\sqrt{\operatorname{det} \sigma^{(i-1)}}}{\sqrt{\operatorname{det} \sigma^{(i)}}}=$ const with the required accuracy (Fig. 7 present the scheme of calculation). Note that the number of iterations depends on geometry of the sample and components of the conductivity tensor. Fig. 8 illustrates the dependence of variation of a relative error on the number of iterations in the samples of $2 \times 1$ and $3 \times 1$ dimension. It is customary here that the ratio of the main summands of the sample conductivity tensor is $\sigma_{1} / \sigma_{2}=3$, and the curves correspond to the various values of the angle $\theta$ (see (3.1)). As we can see from these figures, after just $2-3$ iterations, the error stabilizes and does not exceed $1.6 \%$.

However, the size of error $\delta$ is mainly determined by the length of the central contact $c(\delta=0$ only in the case where $c=0)$. This fact is demonstrated in Fig. 9 by the curves obtained from the cases, considered in Fig. 5, after 3 iterations.

\section{Discussion of the Results and Conclusions}

The paper deals with various methods for defining an electrical conductivity tensor of an anisotropic medium. Application of the van der Pauw theorem and equation makes the basis of all of them. This equation is well known in the Hall effect theory and is meant 

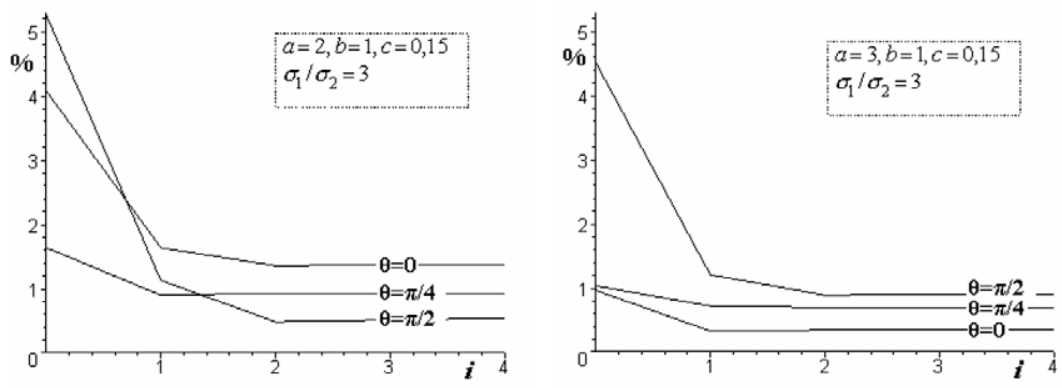

Fig. 8. Dependence of variation of a relative error on the number of iterations.
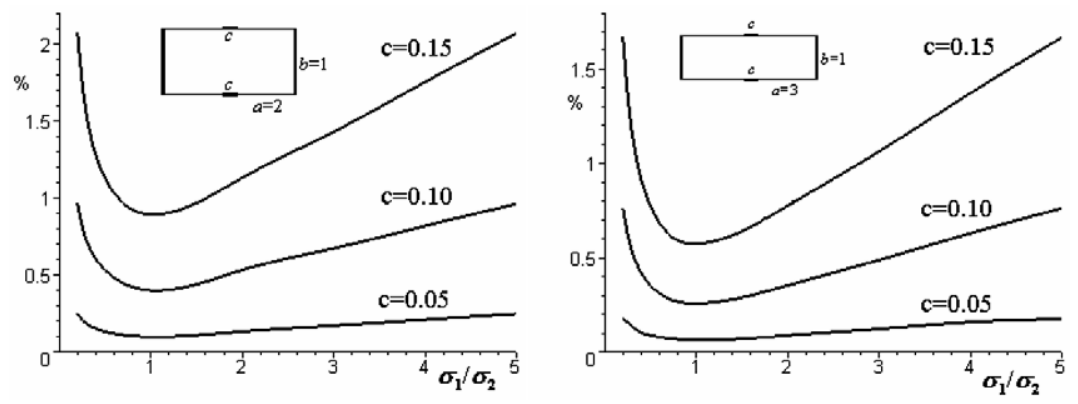

Fig. 9. The values of error $\delta$ after 3 iterations.

for establishing the conductivity of an isotropic medium. Its coefficients are found by measuring differences of potentials and intensity of current.

The novelty of this work lies, first of all, in the statement that, after the affine transformation, one obtains an area in which there is isotropic conductivity and the square of this value is equal to the product of principal components of a tensor (tensor invariant), i.e. , a generalized (Price, 1972) result for the case where the principal directions of a tensor are arbitrarily oriented with respect to the coordinate system used.

It has been proved here that, after taking analogous measurements in an anisotropically conductive sample, the solution to the van der Pauw equation is coincident with the conductivity tensor determinant. If it is known, after making some additional measurements using a rectangular-shaped sample, exact expressions of the conductivity tensor components have been obtained.

With a view to use these expressions, it is necessary to have two samples made from the same substance. Therefore, the algorithms for approximate calculation of the conductivity tensor an a priori error estimation have been presented in the work, using only one rectangular-shaped sample.

Analyzing the errors of the methods developed, it is obvious that they depend upon the extension of the sample form (the ratio $a / b$ of length of the rectangle sides) and on anisotropy of the medium (the ratio $\sigma_{1} / \sigma_{2}$ between the main components of the conductivity tensor), i.e., errors are decreasing with an increase of extension $a / b$ and approaching of the ratio $\sigma_{1} / \sigma_{2}$ to a unit. 
The paper presents a graph of relative errors of values in the most frequent cases where $a / b=2.3$ and $0,2 \leqslant \sigma_{1} / \sigma_{2} \leqslant 5$, while the length of central contacts amounts to $15 \%$ of the length of boundary contacts. It is evident from these graphs that, in all the cases indicated, relative errors of the method proposed do not exceed $1.8 \%$. The errors of this level are acceptable for the majority of physical measurements.

Calculation is performed with the aid of mathematical package Maple. The time of calculating the conductivity tensor takes up 2-6 seconds.

\section{References}

Baronas, R., F. Ivanauskas, M. Sapagovas (2002). Reliability of one dimensional model of moisture diffusion in wood. Informatica, 13(4), 405-416.

Futamata, M. (1992). A computer-controlled measurement for electrical conductivity using the van der Pauw method at various temperatures. Meas. Sci. Technol., 3, 919-921.

Gelder, A.P. van (1995). A new method for measuring the conductivity of arbitrary shaped two-dimensional samples. Phys. B, 204, 149-152.

Kleiza, V., J. Kleiza (1992). Dokl. Akad. Nauk SSSR (Sov. Phys Dokl., 325), 711-715 (in Russian).

Pauw, van der, L.J. (1958). A method of measuring specific resistivity and Hall effect of discs of arbitrary shape. Philips Res. Repts., 13, 1-9.

Price, W.L.V. (1972). Extension of Van der Pauw's theorem for measuring specific resistivity in discs of arbitrary shape to anisotropic media, J. Phys., D5, 1127-1132.

Ramadan, A.A., R.D. Gould, A. Ashour (1994). On the van der Pauw method of resistivity measurements. Thin Solid Films, 239, 272-275.

Rietveld, G. et al. (2003). DC conductivity measurements in the van der Pauw geometry. IEEE Transactions on Instrumentation and Measurement, 52, 449-453.

Qiu, H. et al. (2002). Effect of deposition rate on structural and electrical properties of Al films deposited on glass by electron beam evaporation. Thin Solid Films, 414, 150-153.

Žilinskas, R., V. Kleiza, J. Kleiza (1982). Investigation of the mathematical model of the tranverse voltage phenomenon. Differential Equations and their Application, 31, 41-61 (in Russian). 
J. Kleiza graduated from the Vilnius University in 1969. He received the doctoral degree in mathematics $(\mathrm{PhD})$ from Institute of Mathematics, Minsk in 1975. Currently he is assistant professor of the Department of Mathematical Modelling at the Vilnius Technical University. The main field of scientific interest is the numerical methods for PDE inverse problems as well as mathematical ecology.

M. Sapagovas graduated from the Vilnius University in 1961. He received the doctoral degree in mathematics (PhD) at the Institute of Mathematics in Kiev in 1965. In 1986 he received the doctor habilius degree from the M. Keldysh Institute of Applied Mathematics in Moscow. M. Sapagovas is a professor (1989), corresponding member of the Lithuanian Academy of Sciences (1987), the head of the Department of Numerical Methods at the Institute of Mathematics and Informatics and a professor of the Department of Mathematics and Statistics at the Vytautas Magnus University (2000). The main field of scientific interest is the numerical methods for nonlinear PDE as well as mathematical modelling.

V. Kleiza graduated from the Vilnius University in 1961. He received the doctoral degree in mathematics $(\mathrm{PhD})$ from Steklov Mathematical Institute, Sankt-Petersburg division of Russian Academy of Science, in 1972. Currently he is senior researcher at the Numerical Methods Department of the Institute of Mathematics and Informatics and professor, head of Physical Sciences Department of Kaunas University of Technology. His research interests include Monte Carlo methods, numerical methods for solving nonlinear PDE and mathematical modelling in solid state physics.

\title{
Van Der Pauw metodo taikymas anizotropinei terpei
}

\author{
Jonas KLEIZA, Mifodijus SAPAGOVAS, Vytautas KLEIZA
}

Pasiūlytas anizotropiškai laidžios terpès savitojo laidumo tenzoriaus matavimo metodas, pasižymintis fizikinių matavimų paprastumu: pakanka pagaminti vienodo storio stačiakampio formos bandinị su pritvirtintais jos kraštinèse keturiais elektrodais ir atlikti ịvairius srovès stiprio ir potencialų skirtumo matavimus. Be to, būtinus skaičiavimus galima atlikti greitai, nesinaudojant sudètinga skaičiavimo technika. Gaunamų rezultatų tikslumas priklauso tik nuo bandinio matmenu ir tenzoriaus komponenčių santykių. 